\title{
SUP-NORM BOUNDS FOR EISENSTEIN SERIES
}

\author{
BINGRONG HUANG AND ZHAO XU
}

\begin{abstract}
The paper deals with establishing bounds for Eisenstein series on congruence quotients of the upper half plane, with control of both the spectral parameter and the level. The key observation in this work is that we exploit better the structure of the amplifier by just supporting on primes for the Eisenstein series, which can use both the analytic method as Young did to get a lower bound for the amplifier and the geometric method as Harcos-Templier did to obtain a more efficient treatment for the counting problem.
\end{abstract}

\section{Contents}

1. Introduction

2. Preliminaries

3. A lower bound for the amplifier

4. Counting lattice points

5. Proof of Theorem 1.1

6. Proof of Theorem 1.2

References

\section{INTRODUCTION}

Bounding the sup-norm of Laplace eigenfunctions on manifolds is a classical problem. We shall establish new bounds for the well-studied modular surface $Y_{0}(q)=$ $\Gamma_{0}(q) \backslash \mathbb{H}$ with its hyperbolic measure.

For Hecke-Maass cuspidal newforms $u_{j}$ of spectral parameter $t_{j}$ with $L^{2}$-normalized, in the spectral aspect, the nontrivial bound is due to Iwaniec-Sarnak [11] (for level $q=1$ ) and Blomer-Holowinsky [3] (for square-free level $q$ ) who established

$$
\left\|u_{j}\right\|_{\infty} \ll_{q, \varepsilon} t_{j}^{5 / 12+\varepsilon}
$$

for any $\varepsilon>0$. In the level aspect, the first non-trivial bound is due to BlomerHolowinsky [3] who proved that

$$
\left\|u_{j}\right\|_{\infty} \ll_{t_{j}} q^{-1 / 37},
$$

Date: August 14, 2018.

Key words and phrases. sup-norm, Eisenstein series, trace formula, amplification.

The first author is supported in part by the project of the National Natural Science Foundation of China (11531008), and the second author is supported by the project of the National Natural Science Foundation of China (11501327). 
for square-free $q$. Then this is improved by Templier [13], Harcos-Templier [7, 8], and the current best result is

$$
\left\|u_{j}\right\|_{\infty} \ll_{t_{j}, \varepsilon} q^{-1 / 6+\varepsilon}
$$

Hybrid bounds save a power simultaneously in the spectral and level aspects. The following hybrid bound is established by Blomer-Holowinsky [3.

$$
\left\|u_{j}\right\|_{\infty} \ll t_{j}^{1 / 2}\left(t_{j} q\right)^{-1 / 2300},
$$

for square-free level $q$. In [14, Templier obtains the following hybrid bound which generalizes the best known bounds in the spectral and level aspects simultaneously

$$
\left\|u_{j}\right\|_{\infty} \ll t_{j}^{5 / 12+\varepsilon} q^{-1 / 6+\varepsilon}
$$

for square-free level $q$.

It is natural to consider this problem for the continuous spectrum situation. It seems to have been neglected until Young [15, theorem 1.1] establishes that for $\Omega$ a fixed compact subset of $\mathbb{H}$, and $T \geq 1$,

$$
\max _{z \in \Omega}|E(z, 1 / 2+i T)| \ll_{\Omega, \varepsilon} T^{3 / 8+\varepsilon},
$$

where $E(z, s)$ is the usual real-analytic Eisenstein series for the group $P S L_{2}(\mathbb{Z})$. The Eisenstein series case is similar in some ways to the cuspidal case, but has some technical problems because of the constant term in the Fourier expansion. Let

$$
F(z, s)=E(z, s)-y^{s}-\varphi(s) y^{1-s},
$$

where

$$
\varphi(s)=\xi(2(1-s)) / \xi(2 s)
$$

with

$$
\xi(s)=\pi^{-s / 2} \Gamma(s / 2) \zeta(s) .
$$

Although not stated explicitly in his main theorem, in [15, Section 6], Young actually derived that, for $z=x+i y \in \mathbb{H}$,

$$
F(z, 1 / 2+i T) \ll \begin{cases}T^{3 / 8+\varepsilon}, & \text { if } 1 \ll y \ll T^{1 / 8} \text { or } y \gg T^{1 / 4}, \\ y^{1 / 3} T^{1 / 3+\varepsilon}, & \text { if } T^{1 / 8} \ll y \ll T^{1 / 5} \\ (T / y)^{1 / 2} \log ^{2} T, & \text { if } T^{1 / 5} \ll y \ll T^{1 / 4} .\end{cases}
$$

The main impetus in Young's result is the realization that one can choose an efficient amplifier for the Eisenstein series, which leads to the improved exponent compared to the cusp form case (see [11, Remark 1.6]).

In this paper, we will give the bounds for the Eisenstein series in a wider range of $y$, and then extend to bound the Eisenstein series with square-free levels. Our first main theorem is a generalization of Young's result.

Theorem 1.1. Let $T \geq 1$. For any $z \in \mathbb{H}$, we have

$$
E(z, 1 / 2+i T)=y^{1 / 2+i T}+\varphi(1 / 2+i T) y^{1 / 2-i T}+O\left(y^{-1 / 2}+T^{3 / 8+\varepsilon}\right) .
$$


Remark 1. This result should be compared with Young's result (1.6). We actually get

$$
E(z, 1 / 2+i T) \ll T^{3 / 8+\varepsilon},
$$

provided that $T^{-3 / 4-\varepsilon} \ll y \ll T^{3 / 4+\varepsilon}$, which is a wider range.

Now we turn to consider the Eisenstein series of level $q$, where $q$ is a positive squarefree integer. Let $E_{\mathfrak{a}}(z, s)$ be the Eisenstein series for the congruence group $\Gamma_{0}(q)$ (see \2.1), where $\mathfrak{a}$ is a cusp of $\Gamma_{0}(q)$. Our main result in this case is the following theorem.

Theorem 1.2. Let $z=x+i y \in \mathbb{H}, q$ be a positive square-free integer. Then for $T \geq 1$, we have

$$
E_{\mathfrak{a}}(z, 1 / 2+i T)=\delta_{\mathfrak{a}} y^{1 / 2+i T}+\varphi_{\mathfrak{a}}(1 / 2+i T) y^{1 / 2-i T}+O\left(q^{-1 / 2+\varepsilon}\left(y^{-1 / 2}+T^{3 / 8+\varepsilon}\right)\right) .
$$

In particular, if $\mathfrak{a} \sim \infty$, for all $y \gg 1 / q$, we have

$$
E_{\mathfrak{a}}(z, 1 / 2+i T)=y^{1 / 2+i T}+\varphi_{\mathfrak{a}}(1 / 2+i T) y^{1 / 2-i T}+O\left(q^{-1 / 2+\varepsilon} T^{3 / 8+\varepsilon}\right) .
$$

As in Iwaniec-Sarnak's work [11], we shall use the amplification method which relates the sup-norm problem to an interesting lattice point counting. The key observation in this work is that we exploit better the structure of the amplifier by just supporting on primes for the Eisenstein series, which can use both the analytic method as Young [15] did to get a lower bound for the amplifier and the geometric method as Harcos-Templier [8] and Templier [14] did to obtain a more efficient treatment for the counting problem.

Remark 2. We may compare the error term in (1.13) with the current result of HeckeMaass cusp forms in (1.5). we can see that improved exponents both in spectral aspect and level aspect. This is done because of the choice of the amplifier, and the fact that all Eisenstein series of square-free level are oldforms coming from the full level Eisenstein series. However, by our construction of amplifier, one may expect that

$$
\left\|u_{j}\right\|_{\infty} \ll t_{j}^{3 / 8+\varepsilon} q^{-1 / 4+\varepsilon}
$$

if we assume

$$
\sum_{p \sim N}\left|\lambda_{j}(p)\right|^{2} \gg_{\varepsilon}\left(q t_{j}\right)^{-\varepsilon} N^{1-\varepsilon},
$$

as [11, Remark 1.6] did.

Remark 3. For Hecke-Maass cuspidal newforms, one can restrict the sup-norm problem to the region $z \in \mathcal{F}(q)$ (see Definition 2.1) by the Atkin-Lehner theory which tells us that each Hecke-Maass cuspidal newform is an eigenfunction of the corresponding Atkin-Lehner operators. When it comes to Eisenstein series, we can replace the Atkin-Lehner theory for Hecke-Maass cuspidal newforms by explicit computations on Eisenstein series (see (2.13) and (2.25) $)$. So it is natural to consider the case $y \gg 1 / q$ (see $₫ 2.2)$.

We state that, throughout the paper, $\varepsilon$ is an arbitrarily small positive number which may not be the same in each occurrence. The paper is organized as follows. Section 2 is devoted to the background on Eisenstein series, establishment of the first reduction of Theorem 1.1, and the construction of our amplifier. Bounds via Fourier expansion and a pointwise bound for Eisenstein series via its integral are also summarized in 
Section 2, which is proved by Young [15, Section 4]. In Section 3, a lower bound for our amplifier is established. And then after a summary of the results on counting lattice points from Templier [14] in Section 4, we complete the proof of Theorem 1.1 in Section 5. In the last section, we give the proof of Theorem 1.2.

\section{Preliminaries}

In this section, we collect those basic facts which are needed in this paper. We restrict $q$ to be a positive square-free integer. Let $\mathbb{H}$ be the upper half-plane, $\Gamma=$ $S L_{2}(\mathbb{Z})$ the full modular group, and $\Gamma_{0}(q)$ the Hecke congruence group of level $q$.

Let $\mathcal{A}(\Gamma \backslash \mathbb{H})$ denote the space of automouphic functions of weight zero, i.e., the functions $f: \mathbb{H} \rightarrow \mathbb{C}$ which are $\Gamma$-periodic. Let $\mathcal{L}(\Gamma \backslash \mathbb{H})$ denote the subspace of square-integrable functions with respect to the inner product

$$
\langle f, g\rangle=\int_{\Gamma \backslash \mathbb{H}} f(z) \overline{g(z)} d \mu z
$$

where $d \mu z=y^{-2} d x d y$ is the invariant measure on $\mathbb{H}$. The Laplace operator

$$
\Delta=-y^{2}\left(\frac{\partial^{2}}{\partial x^{2}}+\frac{\partial^{2}}{\partial y^{2}}\right)
$$

acts in the dense subspace of smooth functions in $\mathcal{L}(\Gamma \backslash \mathbb{H})$ such that $f$ and $\Delta f$ are both bounded; it has a self-adjoint extension which yields the spectral decomposition $\mathcal{L}(\Gamma \backslash \mathbb{H})=\mathbb{C} \oplus \mathcal{C}(\Gamma \backslash \mathbb{H}) \oplus \mathcal{E}(\Gamma \backslash \mathbb{H})$. Here $\mathbb{C}$ is the space of constant functions, $\mathcal{C}(\Gamma \backslash \mathbb{H})$ is the space of cusp forms and $\mathcal{E}(\Gamma \backslash \mathbb{H})$ is the space of Eisenstein series. We will focus on $\mathcal{E}(\Gamma \backslash \mathbb{H})$.

2.1. Eisenstein series for $\Gamma$ and $\Gamma_{0}(q)$. The Eisenstein series $E(z, s)$ for $\Gamma$ is defined as

$$
E(z, s)=\sum_{\gamma \in \Gamma_{\infty} \backslash \Gamma}(\operatorname{Im} \gamma z)^{s}
$$

if $\operatorname{Re}(s)>1$, and then by analytic continuation for all $s \in \mathbb{C}$. Here $\Gamma_{\infty}$ is the stability group of $\infty$. For any integer $\ell \geq 1$, the Hecke operator $T_{\ell}$ is defined by

$$
\left(T_{\ell} f\right)(z)=\frac{1}{\sqrt{\ell}} \sum_{a d=\ell} \sum_{b(\bmod d)} f\left(\frac{a z+b}{d}\right) .
$$

Moreover, the reflection operator $R$ defined by $(R f)(z)=f(-\bar{z}) . \quad f$ is called even or odd according to $(R f)(z)=f(z)$ or $(R f)(z)=-f(z)$. All the Eisenstein series $E(z, s)$ are even and they are eigenfunctions of the Hecke operators

$$
T_{\ell} E(z, s)=\eta(\ell, s) E(z, s)
$$

where

$$
\eta(\ell, s)=\sum_{a d=\ell}(a / d)^{s-1 / 2} .
$$

We will write $\eta_{i t}(\ell)=\eta(\ell, 1 / 2+i t)$. And for any $m, n \in \mathbb{Z}_{>0}$, they enjoy the Hecke relation:

$$
\eta_{i t}(m) \eta_{i t}(n)=\sum_{d \mid(m, n)} \eta_{i t}\left(\frac{m n}{d^{2}}\right) .
$$


We have the Fourier expansion

$$
E(z, s)=y^{s}+\varphi(s) y^{1-s}+\frac{2 \sqrt{y}}{\xi(2 s)} \sum_{n \neq 0} \eta(|n|, s-1 / 2) K_{s-1 / 2}(2 \pi|n| y) e(n x) .
$$

Now we consider the Eisenstein series for $\Gamma_{0}(q)$, and we collect several statements about them from [4]. The Eisenstein series $E_{\mathfrak{a}}(z, s)$ for $\Gamma_{0}(q)$ at a cusp $\mathfrak{a}$ is defined as

$$
E_{\mathfrak{a}}(z, s)=\sum_{\gamma \in \Gamma_{\mathfrak{a}} \backslash \Gamma_{0}(q)}\left(\operatorname{Im} \sigma_{\mathfrak{a}}^{-1} \gamma z\right)^{s},
$$

if $\operatorname{Re}(s)>1$ and by analytic continuation for all $s \in \mathbb{C}$. Here $\Gamma_{\mathfrak{a}}$ is the stability group of $\mathfrak{a}$ and $\sigma_{\mathfrak{a}} \in S L_{2}(\mathbb{R})$ such that $\sigma_{\mathfrak{a}} \infty=\mathfrak{a}$ and $\sigma_{\mathfrak{a}}^{-1} \Gamma_{\mathfrak{a}} \sigma_{\mathfrak{a}}=\Gamma_{\infty}$. The scaling matrix $\sigma_{\mathfrak{a}}$ is only determined up to a translation from the right; however the Eisenstein series does not depend on the choice of $\sigma_{\mathfrak{a}}$, not even on the choice of a cusp in the equivalence class. We give some explicit computations on Eisenstein series. We use the details on [5]. Recall that $q$ is square-free, so every cusp of $\Gamma_{0}(q)$ is equivalent to $\mathfrak{a}=1 / v$ with $v \mid q$. The complementary divisor $w=q / v$ is the width of $\mathfrak{a}$. (In the case $w=1$, so $v=q$, which means $\mathfrak{a} \sim \infty$.) Let $\overline{\mathfrak{a}}$ stand for the cusp "dual" to $\mathfrak{a}$ in the sense that $\overline{\mathfrak{a}} \sim 1 / w$ if $\mathfrak{a} \sim 1 / v$. The scaling matrix of $\mathfrak{a}$ can be chosen as

$$
\sigma_{\mathfrak{a}}=\left(\begin{array}{cc}
\sqrt{w} & 0 \\
v \sqrt{w} & 1 / \sqrt{w}
\end{array}\right)
$$

Thus, we have

$$
\sigma_{\mathfrak{a}}^{-1} \Gamma_{0}(q)=\left\{\left(\begin{array}{cc}
a / \sqrt{w} & b / \sqrt{w} \\
c \sqrt{w} & d \sqrt{w}
\end{array}\right):\left(\begin{array}{ll}
a & b \\
c & d
\end{array}\right) \in S L_{2}(\mathbb{Z}), c+a v \equiv 0(v w)\right\} .
$$

The coset $\Gamma_{\infty} \backslash \sigma_{\mathfrak{a}}^{-1} \Gamma_{0}(q)$ is parametrized by pairs of integers $(c, d)=1, c \equiv 0(\bmod v)$ and $(c / v, w)=1$. Therefore the Eisenstein series for the cusp $\mathfrak{a} \sim 1 / v$ is given by

$$
E_{\mathfrak{a}}(z, s)=\frac{1}{2}\left(\frac{y}{w}\right)^{s} \sum_{c}^{*} \sum_{d}|c v z+d|^{-2 s},
$$

where $*$ means the summation is over $(c, d) \in \mathbb{Z}^{2}$ with $(c, d)=1,(c, w)=1$ and $(v, d)=1$. Then, by trivial computation, we have

$$
\begin{aligned}
E_{\mathfrak{a}}\left(\frac{-1}{q z}, s\right) & =\frac{1}{2}\left(\frac{y}{q|z|^{2} w}\right)^{s} \sum_{c}^{*} \sum_{d}\left|-\frac{c v}{q z}+d\right|^{-2 s} \\
& =\frac{1}{2}\left(\frac{y}{v}\right)^{s} \sum_{c} \sum_{d}^{*}|d w z-c|^{-2 s} \\
& =E_{\overline{\mathfrak{a}}}(z, s) .
\end{aligned}
$$

And by [4, Eq. (3.25)], we have the following explicit relation between $E_{\mathfrak{a}}(z, s)$ and $E(z, s)$,

$$
E_{\mathfrak{a}}(z, s)=\zeta_{q}(2 s) \mu(v)(q v)^{-s} \sum_{\beta \mid v} \sum_{\gamma \mid w} \mu(\beta \gamma) \beta^{s} \gamma^{-s} E(\beta \gamma z, s)
$$

where

$$
\zeta_{q}(s)=\prod_{p \mid q}\left(1-p^{-s}\right)^{-1}
$$


The Fourier expansion of the Eisenstein series at cusp $\mathfrak{a}$ is known ([9, Theorem $3.4])$

$$
E_{\mathfrak{a}}(z, s)=\delta_{\mathfrak{a}} y^{s}+\varphi_{\mathfrak{a}}(s) y^{1-s}+\sum_{n \neq 0} \varphi_{\mathfrak{a}}(n, s) W_{s}(n z),
$$

where $\delta_{\mathfrak{a}}=1$ if $\mathfrak{a} \sim \infty$ or $\delta_{\mathfrak{a}}=0$ otherwise, and

$$
\begin{gathered}
\varphi_{\mathfrak{a}}(s)=\pi^{1 / 2} \frac{\Gamma(s-1 / 2)}{\Gamma(s)} \sum_{c} \frac{S_{\mathfrak{a}}(0,0 ; c)}{c^{2 s}} \\
\varphi_{\mathfrak{a}}(n, s)=\pi^{s} \Gamma(s)^{-1}|n|^{s-1} \sum_{c} \frac{S_{\mathfrak{a}}(0, n ; c)}{c^{2 s}},
\end{gathered}
$$

with $S_{\mathfrak{a}}(0, n ; c)=S_{\mathfrak{a} \infty}(0, n ; c)$ and

$$
\begin{aligned}
& S_{\mathfrak{a} \mathfrak{b}}(m, n ; c):=\sum_{\left(\begin{array}{l}
a * \\
c
\end{array}\right) \in B \backslash \sigma_{\mathfrak{a}}^{-1} \Gamma \sigma_{\mathfrak{b}} / B} e\left(\frac{m d+n a}{c}\right), \\
& B=\left\{\left(\begin{array}{ll}
1 & b \\
0 & 1
\end{array}\right): b \in \mathbb{Z}\right\}
\end{aligned}
$$

and $W_{s}(z)$ is the Whittaker function given by

$$
W_{s}(n z)=2 \sqrt{|n| y} K_{s-1 / 2}(2 \pi|n| y) e(n x) .
$$

Now, let us end this subsection with a very rough introduction on Hecke-Maass cuspidal forms for $\Gamma$. Let $\left\{u_{j}\right\}$ be an orthonormal basis of the space of Maass cusp forms for $\Gamma$ such that $\Delta u_{j}(z)=\lambda_{j} u_{j}(z)$ and $T_{n} u_{j}(z)=\lambda_{j}(n) u_{j}(z)$. The Hecke eigenvalues $\lambda_{j}(n)$ also enjoy the Hecke relation:

$$
\lambda_{j}(m) \lambda_{j}(n)=\sum_{d \mid(m, n)} \lambda_{j}\left(\frac{m n}{d^{2}}\right)
$$

2.2. Atkin-Lehner operators and a gap principle. We let $G L_{2}(\mathbb{R})^{+}$act on the upper-half plane $\mathbb{H}$ by the usual fractional linear transformations. If $q$ is square-free, for each divisor $d \mid q$, we consider the matrices $W_{d} \in M_{2}(\mathbb{Z})$ of determinant $d$ such that

$$
W_{d} \equiv\left(\begin{array}{ll}
* & * \\
0 & *
\end{array}\right) \quad(\bmod q) \text { and } W_{d} \equiv\left(\begin{array}{ll}
0 & * \\
0 & 0
\end{array}\right) \quad(\bmod d) .
$$

Scaling the $W_{d}$ 's by $1 / \sqrt{d}$ we obtain matrices in $S L_{2}(\mathbb{R})$ which is called the AtkinLehner operators. The Atkin-Lehner operators together for all $d \mid q$ form a subgroup $A_{0}(q)$ of $S L_{2}(\mathbb{R})$ containing $\Gamma_{0}(q)$ as a normal subgroup. The quotient group $A_{0}(q) / \Gamma_{0}(q)$ is isomorphic to $(\mathbb{Z} / 2 \mathbb{Z})^{\omega(q)}$, where $\omega(q)$ is the number of distinct prime factors of $q$.

Definition 2.1. Let $\mathcal{F}(q)$ be the set of $z \in \mathbb{H}$ such that $\operatorname{Im}(z) \geq \operatorname{Im}(A z)$ for all $A \in A_{0}(q)$, which is a fundamental domain for $A_{0}(q)$.

For $q$ being prime, it is easy to see that $A_{0}(q)=\Gamma_{0}(q) \cup A_{q} \Gamma_{0}(q)$, where

$$
A_{q}=\frac{1}{\sqrt{q}}\left(\begin{array}{l}
r^{-1} \\
q
\end{array}\right)
$$


By the definition of $E_{\mathfrak{a}}(z, s)$, we have $E_{\mathfrak{a}}(\gamma z, s)=E_{\mathfrak{a}}(z, s)$, if $\gamma \in \Gamma_{0}(q)$. And by (2.13), we have

$$
A_{q} E_{\mathfrak{a}}(z, s)=E_{\mathfrak{a}}\left(\frac{-1}{q z}, s\right)=E_{\overline{\mathfrak{a}}}(z, s) .
$$

Hence for $A \in A_{0}(q)$, we have $E_{\mathfrak{a}}(A z, s)=E_{\mathfrak{b}}(z, s)$, where $\mathfrak{b}=\mathfrak{a}$ if $A \in \Gamma_{0}(q)$, and $\mathfrak{b}=\overline{\mathfrak{a}}$ if $A \in A_{q} \Gamma_{0}(q)$.

For Hecke-Maass cuspidal newform $u_{j}$, by Atkin-Lehner theory we know that it is an eigenvector for the Atkin-Lehner operators with eigenvalues \pm 1 . Therefore, we may assume that $z \in \mathcal{F}(q)$ when investigating the sup-norm of a Hecke-Maass cuspidal newform. By (2.25), we can still make the same assumption for Eisenstein series, that is, $z \in \mathcal{F}(q)$. Note that for $z \in \mathcal{F}(q)$, by [7, Lemma 2.2], we have

$$
\operatorname{Im} z \geq \frac{\sqrt{3}}{2 q}
$$

and

$$
|c z+d|^{2} \geq 1 / q
$$

where $(c, d) \in \mathbb{Z}^{2}$ is distinct from $(0,0)$.

2.3. Amplified pre-trace formula. Let $k \in \mathcal{C}^{\infty}([0, \infty))$ with rapid decay. Then, it can be viewed as the inverse of the Selberg transform of a function $h(t)$, which is given by the following three steps (see [9, (1.64)]:

$$
\begin{aligned}
g(\xi) & :=\frac{1}{2 \pi i} \int_{-\infty}^{\infty} e^{-i r \xi} h(r) d r, \\
2 q(v) & :=g(2 \log (\sqrt{v+1}+\sqrt{v})), \\
k(u) & :=-\frac{1}{\pi} \int_{u / 4}^{\infty}(v-u / 4)^{-1 / 2} d q(v) .
\end{aligned}
$$

Assume now that $k(z, w)=k(u(z, w))$ is a point point-pair invariant kernel with

$$
u(z, w):=\frac{|z-w|^{2}}{\operatorname{Im}(z) \operatorname{Im}(w)}, \quad z, w \in \mathbb{H},
$$

and $h(t)$ is the corresponding Selberg transform which satisfies the conditions (see [9, $(1.63)])$

$$
\left\{\begin{array}{l}
h(t) \text { is even, } \\
h(t) \text { is holomorphic in the strip }|\operatorname{Im} t|<\frac{1}{2}+\varepsilon, \\
h(t) \ll(|t|+1)^{-2-\varepsilon} \text { in the strip. }
\end{array}\right.
$$

Due to [9, Theorem 7.4], we have the spectral expansion

$$
\begin{aligned}
K(z, w): & =\sum_{\gamma \in \Gamma} k(\gamma z, w) \\
& =\sum_{j \geq 0} h\left(t_{j}\right) u_{j}(z) \overline{u_{j}(w)}+\frac{1}{4 \pi} \int_{-\infty}^{\infty} h(r) E(z, 1 / 2+i r) \overline{E(w, 1 / 2+i r)} d r .
\end{aligned}
$$


Then it follows by applying the $\ell$ th Hecke operator on the spectral expansion that (cf. displays (1.3) and (1.4) in [11])

$$
\begin{aligned}
& \frac{1}{\sqrt{\ell}} \sum_{\gamma \in \mathcal{M}(\ell)} k(\gamma z, z) \\
= & \sum_{j \geq 0} \lambda_{j}(\ell) h\left(t_{j}\right)\left|u_{j}(z)\right|^{2}+\frac{1}{4 \pi} \int_{-\infty}^{\infty} \eta_{i r}(\ell) h(r)|E(z, 1 / 2+i r)|^{2} d r,
\end{aligned}
$$

where $\mathcal{M}(\ell)$ is the set of matrices $\gamma=\left(\begin{array}{ll}a & b \\ c & d\end{array}\right) \in M_{2}(\mathbb{Z})$ with $\operatorname{det}(\gamma)=\ell$.

Now we consider the following sum

$$
\sum_{j \geq 0} h\left(t_{j}\right) A_{j}\left|u_{j}(z)\right|^{2}+\frac{1}{4 \pi} \int_{-\infty}^{\infty} h(r) A_{i r}|E(z, 1 / 2+i r)|^{2} d r
$$

where

$$
A_{j}=\left|\sum_{n} x_{n} \lambda_{j}(n)\right|^{2}, \quad A_{i r}=\left|\sum_{n} x_{n} \eta_{i r}(n)\right|^{2}
$$

and $h: \mathbb{R} \cup[-i / 2, i / 2] \rightarrow \mathbb{R}_{+}$is a positive even smooth function of rapid decay, $\left(x_{n}\right)$ is sequence of complex numbers supported on finitely many $n$ 's. After squaring out the $n$-sum and applying the Hecke relations (2.7) and (2.22), we arrive at

$$
\left.\sum_{\ell}\left|y_{\ell}\right|\left|\sum_{j \geq 0} \lambda_{j}(\ell) h\left(t_{j}\right)\right| u_{j}(z)\right|^{2}+\frac{1}{4 \pi} \int_{-\infty}^{\infty} \eta_{i r}(\ell) h(r)|E(z, 1 / 2+i r)|^{2} d r \mid,
$$

where

$$
y_{\ell}:=\sum_{\substack{d \mid(m, n) \\ \ell=m n / d^{2}}} x_{m} \overline{x_{n}}=\sum_{\substack{d \geq 1 \\ \ell=\ell_{1} \ell_{2}}} x_{d \ell_{1}} \overline{x_{d \ell_{2}}} .
$$

By (2.28), it follows that

$$
\begin{aligned}
& \sum_{j \geq 0} h\left(t_{j}\right) A_{j}\left|u_{j}(z)\right|^{2}+\frac{1}{4 \pi} \int_{-\infty}^{\infty} h(r) A_{i r}|E(z, 1 / 2+i r)|^{2} d r \\
= & \sum_{\ell} \frac{\left|y_{\ell}\right|}{\sqrt{\ell}} \sum_{\gamma \in \mathcal{M}(\ell)}|k(\gamma z, z)| .
\end{aligned}
$$

This identity is what we call the amplified pre-trace formula.

To obtain upper bounds we use a test function $h(r)$ which is localized for $r$ near $T$, with $T \geq 2$ being a parameter. We need a suitable point-pair kernel and the coming estimate.

Lemma 2.1. For all $T \geq 1$, there is a point-pair kernel $k_{T} \in C_{c}^{\infty}([0, \infty))$, supported on $[0,1]$, which satisfies the following properties:

(i) The spherical transform $h_{T}(r)$ is positive for all $r \in \mathbb{R} \cup i \mathbb{R}$,

(ii) For all $T \leq r \leq T+1, h_{T}(r) \gg 1$,

(iii) For all $u \geq 0,\left|k_{T}(u)\right| \leq T$,

(iv) For all $T^{-2} \leq u \leq 1,\left|k_{T}(u)\right| \leq \frac{T^{1 / 2}}{u^{1 / 4}}$.

Proof. See Templier [14, Lemma 2.1]. 
Lemma 2.2. Let $k_{T}$ be as in the above lemma. Let $M:[0,1] \rightarrow \mathbb{R}_{+}$be a nondecreasing function with finitely many discontinuities such that $M(\delta) \ll \delta^{\alpha}$ for some $\alpha>0$. Then the following holds with $\beta:=\max (1 / 2,1-2 \alpha)$ :

$$
\int_{0}^{1}\left|k_{T}(\delta)\right| d M(\delta) \ll T^{\beta}
$$

Proof. See Templier [14, Lemma 2.3].

Hence, by (2.31), we have

$$
\int_{T}^{T+1} A_{i r}|E(z, 1 / 2+i r)|^{2} d r \ll \sum_{\ell} \frac{\left|y_{\ell}\right|}{\sqrt{\ell}} \sum_{\gamma \in \mathcal{M}(\ell)}\left|k_{T}(\gamma z, z)\right| .
$$

And now, we have three main problems to overcome to obtain a bound for $E(z, 1 / 2+$ $i t)$. The first problem is to relate a pointwise bound on $E(z, 1 / 2+i t)$ to an integral bound of the type occurring in (2.32). This has done by Young [15], see Lemma 2.4 below. The second problem is to choose $x_{n}$ in order to make the amplifier to be large on an integral of $t$ 's of length $T^{-\varepsilon}$, but not simply be large at a single value of $t$. The last problem is to give a good upper bound for the the right-hand side of (2.32), that is, to count lattice points efficiently.

2.4. Summary of Young's results. We need the following modified version of Young [15].

Lemma 2.3. For $t \geq 1$, and $y \gg 1$, we have

$$
F(z, 1 / 2+i t) \ll(t / y)^{1 / 2} \log ^{2} t+t^{1 / 6+\varepsilon} .
$$

Proof. The proof is similar to [15, Lemma 3.1]. So a sketch proof is enough. By Stirling's formula,

$$
F(z, 1 / 2+i t) \ll \frac{\sqrt{y}}{|\zeta(1+2 i t)|} \sum_{n=1}^{\infty} d(n)\left|K_{i t}(2 \pi n y)\right| \cosh (\pi t / 2) .
$$

We have uniform bounds on the $K$-Bessel function which we extract from the uniform asymptotic expansions due to Balogh [1]:

$$
e^{\frac{\pi}{2} t} K_{i t}(u) \ll \begin{cases}t^{-1 / 4}(t-u)^{-1 / 4}, & \text { if } 0<u<t-C t^{1 / 3}, \\ t^{-1 / 3}, & \text { if }|u-t| \leq C t^{1 / 3}, \\ u^{-1 / 4}(u-t)^{-1 / 4} \exp \left(-c\left(\frac{u}{t}\right)^{3 / 2}\left(\frac{u-t}{t^{1 / 3}}\right)^{3 / 2}\right), & \text { if } u>t+C t^{1 / 3},\end{cases}
$$

where $c$ and $C$ are fixed constants. With the help of this, we can assume $y \ll t$, since the exponential decay of the $K$-Bessel function. The deduction of [15, Lemma 3.1] gives us

$$
F(z, 1 / 2+i t) \ll(t / y)^{1 / 2} \log ^{2} t+y^{1 / 2} t^{-1 / 3+\varepsilon} \ll(t / y)^{1 / 2} \log ^{2} t+t^{1 / 6+\varepsilon} .
$$

We also need the following Lemma of Young [15].

Lemma 2.4. Suppose $y, T \gg 1$. Then

$$
|E(z, 1 / 2+i T)|^{2} \ll y \log ^{6} T+\log ^{5} T \int_{|r| \leq 4 \log T}|E(z, 1 / 2+i T+i r)|^{2} d r .
$$

Proof. See Young [15, Corollary 4.2]. 
2.5. Amplifier. It is convenient to give our amplifier now. We should modify the construction of Young [15]. Let

$$
\mathcal{P}=\{p \text { prime }: N \leq p \leq 2 N\} .
$$

be a large set of primes, and define

$$
x_{n}:= \begin{cases}w(n / N) \log (n) \eta_{i t}(n), & \text { if } n \in \mathcal{P} \\ 0, & \text { otherwise }\end{cases}
$$

where $w$ is a fixed, compactly-support positive function on the positive reals, with

$$
\operatorname{supp}(w) \subset[1,2], \quad 0 \leq w(r) \leq 1, \quad \text { and } \quad \int_{-\infty}^{\infty} w(r) d r \neq 0 .
$$

Hence $y_{\ell}$ defined in (2.30) satisfies:

$$
y_{\ell} \ll \begin{cases}N, & \text { if } \ell=1, \\ \log ^{2} N, & \text { if } \ell=\ell_{1} \ell_{2} \text { with } \ell_{1}, \ell_{2} \in \mathcal{P}, \\ 0, & \text { otherwise. }\end{cases}
$$

The advantage of this amplifier can be seen in Sections $[3$ and 4 ,

\section{A LOWER BOUND FOR THE AMPLIFIER}

Define

$$
A_{N}(t, r)=\sum_{p=2}^{\infty} w(p / N) \log (p) \eta_{i t}(p) \eta_{i r}(p)
$$

where $\eta_{i t}(n)$ is defined in (2.6) and $w$ is a fixed function satisfied to (2.34). Let

$$
L(s)=\frac{\zeta(s+i t+i r) \zeta(s-i t+i r) \zeta(s+i t-i r) \zeta(s-i t-i r)}{\zeta(2 s)},
$$

for $\operatorname{Re}(s)>1$, where $\zeta(s)$ is the Riemann zeta-function. Using a well-known identity of Ramanujan [12, equation (15)] (see also [10, equation (1.28)]), we derive

$$
L(s)=\sum_{n=1}^{\infty} \frac{\eta_{i t}(n) \eta_{i r}(n)}{n^{s}}
$$

Then, by the Euler product of $L(s)$, we have

$$
\begin{aligned}
-\frac{L^{\prime}}{L}(s)= & \sum_{p} \log p\left(\frac{p^{-s-i t-i r}}{1-p^{-s-i t-i r}}+\frac{p^{-s+i t-i r}}{1-p^{-s+i t-i r}}\right. \\
& \left.\quad+\frac{p^{-s-i t+i r}}{1-p^{-s-i t+i r}}+\frac{p^{-s+i t+i r}}{1-p^{-s+i t+i r}}-\frac{2 p^{-2 s}}{1-p^{-2 s}}\right) \\
= & \sum_{n=1}^{\infty} \frac{b(n)}{n^{s}}, \quad \operatorname{Re} s>1,
\end{aligned}
$$

say. By the Taylor expansion, we derive that

$$
b(n)= \begin{cases}\log (p) \eta_{i t}(p) \eta_{i r}(p), & \text { if } n=p \\ \log (p) b_{p, k}, & \text { if } n=p^{k}, \quad k \geq 2 \\ 0, & \text { otherwise }\end{cases}
$$


with $\left|b_{p, k}\right| \leq 6$ for all $p$ prime and $k \geq 2$. Define

$$
B_{N}(t, r)=\sum_{n=1}^{\infty} w(n / N) b(n)
$$

By (2.34) and (3.4), we have

$$
A_{N}(t, r)=B_{N}(t, r)+O(\sqrt{N}) .
$$

So we can estimate $A_{N}(t, r)$ by the estimation of $B_{N}(t, r)$.

Lemma 3.1. Suppose that $\log N \gg(\log T)^{2 / 3+\delta}$, and $t, r=T+O\left((\log N)^{-1-\delta}\right)$, for some fixed $\delta>0$. Then

$$
A_{N}(t, r)=2 N \widetilde{w}(1)(1+o(1)),
$$

where $\widetilde{w}(s)=\int_{0}^{\infty} w(y) y^{s-1} d y$ is the Mellin transform of $w$.

Fouvry, Kowalski, and Michel [6, Lemma 2.4] prove a result with a similar conclusion, but their method requires $N \gg T^{3}$. Young [15, Lemma 5.1] also gives a similar result, but his summation is over all integers. Our proof is analogous to the proof of [15, Lemma 5.1].

Proof. Taking a Mellin transform and by (3.2) and (3.3), we derive

$$
\begin{aligned}
B_{N}(t, r)=\frac{1}{2 \pi i} \int_{(2)} N^{s} \widetilde{w}(s) & \left(-\frac{L^{\prime}}{L}(s)\right) d s \\
=\frac{1}{2 \pi i} \int_{(2)} N^{s} \widetilde{w}(s)( & -\frac{\zeta^{\prime}}{\zeta}(s+i t+i r)-\frac{\zeta^{\prime}}{\zeta}(s-i t+i r) \\
& \left.\quad-\frac{\zeta^{\prime}}{\zeta}(s+i t-i r)-\frac{\zeta^{\prime}}{\zeta}(s-i t-i r)+\frac{\zeta^{\prime}}{\zeta}(2 s)\right) d s .
\end{aligned}
$$

Next we move the contour to the left, to one along the straight line segments $L_{1}, L_{2}, L_{3}$ defined by $L_{1}=\left\{1-\frac{c}{(\log T)^{2 / 3+\delta / 2}}+i \tau:|\tau| \leq 100 T\right\}, L_{2}=\{1+i \tau:|\tau| \geq 100 T\}$, and the short horizontal segments $L_{3}=\left\{\sigma \pm 100 i T: 1-\frac{c}{(\log T)^{2 / 3+\delta / 2}} \leq \sigma \leq 1\right\}$, where $c$ is a small positive number such that $L(s)$ is zero-free on the boundary and right side of $L_{1} \cup L_{2} \cup L_{3}$. By [10, Theorem 8.29], we may also assume the Vinogradov-Korobov bound $\zeta^{\prime}(s) / \zeta(s) \ll(\log |\tau|)^{2 / 3}(\log \log \tau)^{1 / 3}$ in this region. The integrals along the line segments $L_{2}$ and $L_{3}$ are trivially bounded by $O\left(T^{-100}\right)$ by the rapid decay of $\widetilde{w}$. The new line $L_{1}$ gives an amount that is certainly

$$
\ll N(\log T) \exp \left(-\frac{\log N}{(\log T)^{2 / 3+2 \delta / 3}}\right) \ll \frac{N}{(\log T)^{100}} .
$$

Now we need to analyze the residue of the poles. The contribution from $s=$ $1+i t+i r$ and $s=1-i t-i r$ is negligible because of the rapid decay of $\widetilde{w}(s)$. The residue at $s=1+i t-i r$ contributes

$$
R=N^{1+i t-i r} \widetilde{w}(1+i t-i r) .
$$

Write $t=r+\eta$ (by assumption, $\eta=O\left((\log N)^{-1-\delta}\right)$ ), and using Taylor expansions, we have

$$
R=N^{1+i \eta} \widetilde{w}(1+i \eta)=N \widetilde{w}(1)(1+O(|\eta| \log N))=N \widetilde{w}(1)(1+o(1)) .
$$


By a similar argument for the residue at $s=1-i t+i r$, we have

$$
B_{N}(t, r)=2 N \widetilde{w}(1)(1+o(1)) .
$$

Hence, by (3.6)), we prove the lemma.

\section{Counting lattice points}

We need the counting results in Templier [14, where he counted lattice points respect to the level and the parameter. Here, we restrict the level to be 1 .

For $z \in \mathbb{H}, \delta>0$ and the integer $\ell$, let $\mathcal{M}(z, \ell, \delta)$ be the finite set of matrices $\gamma=\left(\begin{array}{ll}a & b \\ c & d\end{array}\right)$ in $M_{2}(\mathbb{Z})$ such that

$$
\operatorname{det}(\gamma)=\ell, \quad u(\gamma z, z) \leq \delta .
$$

Denote by $M=M(z, \ell, \delta)$ its cardinality. We split the counting $M$ of matrices $\gamma=\left(\begin{array}{ll}a & b \\ c & d\end{array}\right)$ as

$$
M=M_{*}+M_{u}+M_{p}
$$

according to whether $c \neq 0$ and $(a+d)^{2} \neq 4 \ell$ (generic), or $c=0$ and $a \neq d$ (uppertriangular), or $(a+d)^{2}=4 \ell$ (parabolic).

Now we should recall some results on counting lattice points in Templier [14].

Lemma 4.1. For any $z=x+i y \in D$, and any integer $L$ and $0<\delta<1$, we have

$$
\sum_{1 \leq \ell \leq L} M_{*}(z, \ell, \delta) \ll L^{\varepsilon}\left(\frac{L}{y}+L^{3 / 2} \delta^{1 / 2}+L^{2} \delta\right) .
$$

Proof. See [14, Lemma 1.3].

Recall that $\mathcal{F}(q)$ is the set of $z \in \mathbb{H}$ such that $\operatorname{Im} z \geq \operatorname{Im} A z$ for all $A \in A_{0}(q)$. The original results of the following two lemmas in Templier [14] were considered in the region $\mathcal{F}(q)$. In our case, the corresponding region $\mathcal{F}(1)$ becomes the well-known fundamental domain of $S L_{2}(\mathbb{Z})$, which is $D=\{z=x+i y \in \mathbb{H}:|x| \leq 1 / 2,|z| \geq 1\}$.

Lemma 4.2. For any $z=x+i y \in D$, and any integer $L$ and $0<\delta<1$, the following estimate holds where $\ell_{1}, \ell_{2}$ run over primes:

$$
\sum_{1 \leq \ell_{1}, \ell_{2} \leq L} M_{u}\left(z, \ell_{1} \ell_{2}, \delta\right) \ll L^{\varepsilon}\left(L+L^{3} \delta^{1 / 2} y\right) .
$$

Proof. See [14, Lemma 4.3].

Remark 4. The original proof of Lemma 4.2 comes from [8, Lemma 4], where three results were stated according to $\ell=\ell_{1} \ell_{2}, \ell=\ell_{1} \ell_{2}^{2}$, or $\ell=\ell_{1}^{2} \ell_{2}^{2}$. We remark that there is an extra condition which states that $L \leq q^{O(1)}$, where $q$ is the level. However, in our case $\left(\ell=\ell_{1} \ell_{2}\right)$, this condition is not needed in the proof. So we remove it here.

Lemma 4.3. For any $z=x+i y \in D$, we have

$$
M_{p}(z, \ell, \delta) \ll\left(1+\ell^{1 / 2} \delta^{1 / 2} y+\ell^{3 / 4} \delta^{3 / 8} y^{-1 / 2}\right) \delta_{\square}(\ell),
$$

where $\delta_{\square}(\ell)=1,0$ depending on whether $\ell$ is a perfect square or not. 
Remark 5. A better bound could be found in [14, Lemma 4.4]. However, in the recent preprint [2, Remark 5], the authors explained that it remained unclear to them to get this better bound. Instead, they provides an alternative bound of a number field version. Here, for completeness, we use their technique of [2, Lemma 13] to give this result which is weaker than [14, Lemma 4.4] but sufficient for us.

Proof. Note that $\ell$ is always a perfect square. Firstly, we consider the case $c=0$. By $(a+d)^{2}=4 \ell=4 a d$, we have $a=d= \pm \ell^{1 / 2}$. So

$$
\delta \geq u(\gamma z, z)=\frac{\left|a z+b-c z^{2}-d z\right|^{2}}{\ell y^{2}}=\frac{b^{2}}{\ell y^{2}} .
$$

Thus, we have $\#(b) \ll 1+\ell^{1 / 2} \delta^{1 / 2} y$. And then, we deduce that the number of possibilities for the parabolic matrix $\gamma=\left(\begin{array}{ll}a & b \\ c & d\end{array}\right)$ with $c=0$ is

$$
\ll\left(1+\ell^{1 / 2} \delta^{1 / 2} y\right) \delta_{\square}(\ell) .
$$

Now we count the number of matrices $\gamma=\left(\begin{array}{ll}a & b \\ c & d\end{array}\right) \in M_{2}(\mathbb{Z})$ with $c \neq 0$ such that

$$
u(\gamma z, z)=\frac{|\gamma z-z|^{2}}{\operatorname{Im}(\gamma z) \operatorname{Im}(z)} \leq \delta,
$$

and $(a+d)^{2}=4 \ell=4(a d-b c)$. We have

$$
\delta \geq u(\gamma z, z) \geq \frac{(\operatorname{Im}(\gamma z-z))^{2}}{\operatorname{Im}(\gamma z) \operatorname{Im}(z)}=\left(\frac{\ell-|c z+d|^{2}}{\ell^{1 / 2}|c z+d|}\right)^{2}=\left(\frac{\ell^{1 / 2}}{|c z+d|}-\frac{|c z+d|}{\ell^{1 / 2}}\right)^{2},
$$

which implies that

$$
|c z+d|=\ell^{1 / 2}\left(1+O\left(\delta^{1 / 2}\right)\right) .
$$

On the other hand, we can also get

$$
\delta \geq u(\gamma z, z)=\frac{\left|a z+b-c z^{2}-d z\right|^{2}}{\ell y^{2}} \geq \frac{\left(\operatorname{Im}\left(a z+b-c z^{2}-d z\right)\right)^{2}}{\ell y^{2}}=\frac{(2 c x-a+d)^{2}}{\ell} .
$$

This deduces that

$$
\frac{|2 c x-a+d|}{\ell^{1 / 2}} \leq \delta^{1 / 2} .
$$

With the help of this and $|a+d|=2 \ell^{1 / 2}$, we obtain

$$
2|| c x+d\left|-\ell^{1 / 2}\right|=|| 2 c x+2 d|-| a+d|| \leq|2 c x-a+d| \leq \ell^{1 / 2} \delta^{1 / 2},
$$

which gives

$$
|c x+d|=\ell^{1 / 2}\left(1+O\left(\delta^{1 / 2}\right)\right) .
$$

We can estimate $c$ by (4.6) and (4.8) as follows:

$$
c^{2} y^{2}=|c z+d|^{2}-|c x+d|^{2}=\ell\left(1+O\left(\delta^{1 / 2}\right)\right)^{2}-\ell\left(1+O\left(\delta^{1 / 2}\right)\right)^{2} \ll \ell \delta^{1 / 2},
$$

and hence

$$
c \ll \frac{\ell^{1 / 2} \delta^{1 / 4}}{y} .
$$


Combining the above together with (4.7) and the identity $(a-d)^{2}+4 b c=0$, we show that (for any fixed $c \neq 0$, we know $a-d$ is divisible by a fixed number at least $c^{1 / 2}$ )

$$
\sharp(c, a-d) \ll \sum_{c \ll \ell^{1 / 2} \delta^{1 / 4} y^{-1}} \frac{c+\ell^{1 / 2} \delta^{1 / 2}}{c^{1 / 2}} \ll \ell^{3 / 4} \delta^{3 / 8} y^{-1 / 2} .
$$

Finally, since the trace $a+d= \pm 2 \ell^{1 / 2}$, the number of possibilities for the parabolic matrix $\gamma=\left(\begin{array}{ll}a & b \\ c & d\end{array}\right)$ with $c \neq 0$ is

$$
\sharp(c, a-d, a+d) \ll \ell^{3 / 4} \delta^{3 / 8} y^{-1 / 2} .
$$

We complete this proof by (4.5) and (4.10).

\section{Proof of Theorem 1.1}

We first suppose $y \gg 1$. By Lemma 2.4, we have that

$$
|E(z, 1 / 2+i T)|^{2} \ll y \log ^{6} T+\log ^{5} T \int_{|r| \leq 4 \log T}|E(z, 1 / 2+i T+i r)|^{2} d r .
$$

On the right hand side above, we dissect the integral into subintervals, each of length $\asymp(\log T)^{-2}$, say. Let $U$ be one of these intervals, and let $t_{U}$ be the left endpoint of $U$. Then by Lemma 3.1, we have

$$
\begin{aligned}
& \int_{r \in U}|E(z, 1 / 2+i T+i r)|^{2} d r \\
\ll & N^{-2} \int_{r \in U}\left|A_{N}\left(T+t_{U}, T+r\right)\right|^{2}|E(z, 1 / 2+i T+i r)|^{2} d r .
\end{aligned}
$$

By (2.32) and (3.1), where $x_{n}$ is defined as in (2.33) with $t=T+t_{U}$, we have

$$
\int_{r \in U}\left|A_{N}\left(T+t_{U}, T+r\right)\right|^{2}|E(z, 1 / 2+i T+i r)|^{2} d r \ll \sum_{\ell} \frac{\left|y_{\ell}\right|}{\sqrt{\ell}} K_{T+t_{U}}(z, \ell),
$$

where $y_{\ell}$ is as in (2.30) and

$$
K_{T}(z, \ell):=\sum_{\gamma \in \mathcal{M}(\ell)}\left|k_{T}(\gamma z, z)\right|=\int_{0}^{1}\left|k_{T}(\delta)\right| d M(z, \ell, \delta) .
$$

Hence we first need to estimate the quantity

$$
A(z, \delta):=\sum_{\ell} \frac{\left|y_{\ell}\right|}{\sqrt{\ell}} M(z, \ell, \delta) .
$$

Since $M=M_{*}+M_{u}+M_{p}$, we decompose $A=A_{*}+A_{u}+A_{p}$ accordingly. By (2.35), we have

$$
A(z, \delta) \ll N M(z, 1, \delta)+\frac{\log ^{2} N}{N} \sum_{\ell_{1}, \ell_{2} \in \mathcal{P}} \sum M\left(z, \ell_{1} \ell_{2}, \delta\right) .
$$

Hence together with the fact that $M_{u}(z, 1, \delta)=0$ and Lemmas 4.1, 4.2 and 4.3, we get the following proposition. 
Proposition 5.1. Let $z=x+i y \in D, 0<\delta<1$. Then

$$
\begin{aligned}
& A_{*}(z, \delta) \ll(N T)^{\varepsilon}\left(N+N^{2} \delta^{1 / 2}+N^{3} \delta\right), \\
& A_{u}(z, \delta) \ll(N T)^{\varepsilon}\left(1+N^{2} \delta^{1 / 2} y\right), \\
& A_{p}(z, \delta) \ll(N T)^{\varepsilon}\left(N+N \delta^{1 / 2} y+N^{3 / 2} \delta^{3 / 8} y^{-1 / 2}\right) .
\end{aligned}
$$

We now execute the integration over $\delta$ in the Stieltjes integral

$$
\sum_{\ell} \frac{\left|y_{\ell}\right|}{\sqrt{\ell}} K_{T}(z, \ell)=\int_{0}^{1}\left|k_{T}(\delta)\right| d A(z, \delta)
$$

From Lemma 2.2 we see that we can make the substitutions $\left(\delta \rightsquigarrow T^{1 / 2}\right),\left(\delta^{1 / 2} \rightsquigarrow T^{1 / 2}\right)$, $\left(\delta^{3 / 8} \rightsquigarrow T^{1 / 2}\right)$ and $(1 \rightsquigarrow T)$ starting from the upper-bound for $A(z, \delta)$ to obtain the bound for the Stieltjes integral as [14, §6] did. Here we emphasize that this formula is used with $T+t_{U}$ in place of $T$, and then one needs to sum up the resulting bounds over the various subintervals $U \subset[-4 \log T, 4 \log T]$. Altogether we obtain from Proposition 5.1 and after some simplifications that:

$$
\begin{aligned}
& \sum_{\ell} \frac{\left|y_{\ell}\right|}{\sqrt{\ell}} K_{T+t_{U}}(z, \ell) \\
& \ll\left(N\left(T+t_{U}\right)\right)^{\varepsilon}\left(N\left(T+t_{U}\right)+N^{3}\left(T+t_{U}\right)^{1 / 2}+N^{2}\left(T+t_{U}\right)^{1 / 2} y\right) \\
& \ll(N T)^{\varepsilon}\left(N T+N^{3} T^{1 / 2}+N^{2} T^{1 / 2} y\right) .
\end{aligned}
$$

From the bound via Fourier expansion in Lemma 2.3, we can assume without loss of generality when establishing Theorem 1.1 that

$$
y \ll T^{1 / 4} .
$$

Combining (5.1), (5.2), (5.3), with (5.7), we obtain that

$$
|E(z, 1 / 2+i T)|^{2} \ll(N T)^{\varepsilon}\left(T N^{-1}+N T^{1 / 2}+T^{1 / 2} y\right)
$$

We choose

$$
N:=T^{1 / 4}
$$

This yields

$$
|E(z, 1 / 2+i T)| \ll T^{3 / 8+\varepsilon},
$$

which implies that

$$
E(z, 1 / 2+i T)=y^{1 / 2+i T}+\varphi(1 / 2+i T) y^{1 / 2-i T}+O\left(T^{3 / 8+\varepsilon}\right),
$$

provided $z \in \bigcup_{k \in \mathbb{Z}}(k+D)$.

Now, we consider the case $z \notin \bigcup_{k \in \mathbb{Z}}(k+D)$. By the definition of fundamental domain, there exists $\gamma=\left(\begin{array}{ll}a & b \\ c & d\end{array}\right) \in S L_{2}(\mathbb{Z})$, such that $\gamma z \in D$. We see that $c \neq 0$, since $\gamma z \notin D$ if $c=0$. So we have

$$
\operatorname{Im} \gamma z \ll \frac{y}{c^{2} y^{2}} \ll y^{-1} .
$$

With the help of (5.10), we get

$$
E(z, 1 / 2+i T)=E(\gamma z, 1 / 2+i T) \ll y^{-1 / 2}+T^{3 / 8+\varepsilon} .
$$


Based on the above arguments, for any $z \in \mathbb{H}$, we obtain

$$
E(z, 1 / 2+i T)=y^{1 / 2+i T}+\varphi(1 / 2+i T) y^{1 / 2-i T}+O\left(y^{-1 / 2}+T^{3 / 8+\varepsilon}\right),
$$

as claimed in Theorem 1.1.

Remark 6. In Young [15, Equation (6.3)], for $y, T \gg 1$, he proved that

$$
|E(z, 1 / 2+i T)|^{2} \ll(N T)^{\varepsilon}\left(T N^{-1}+T^{1 / 2}\left(N+N^{1 / 2} y\right)\right) .
$$

Compared with (5.8), one can see that the term $T^{1 / 2} N^{1 / 2} y$ may be improved to be $T^{1 / 2} y$ by our amplifier and counting method. This is the reason why we can improve Young's result slightly.

\section{Proof of Theorem 1.2}

Let

$$
F_{\mathfrak{a}}(z, s)=E_{\mathfrak{a}}(z, s)-\delta_{\mathfrak{a}} y^{s}-\varphi_{\mathfrak{a}}(s) y^{1-s} .
$$

Then, by (2.8), (2.16), and (2.14), we deduce that

$$
F_{\mathfrak{a}}(z, s)=\zeta_{q}(2 s) \mu(v)(q v)^{-s} \sum_{\beta \mid v} \sum_{\gamma \mid w} \mu(\beta \gamma) \beta^{s} \gamma^{-s} F(\beta \gamma z, s),
$$

where $\mathfrak{a} \sim 1 / v, v \mid q$, and $w=q / v$. Since $q$ is square-free, for $\operatorname{Re}(s)=1$, we have

$$
\left|\zeta_{q}(s)\right| \leq \prod_{p \mid q}\left(1-p^{-1}\right)^{-1}=q \varphi(q)^{-1} \ll \log \log q,
$$

where $\varphi(q)$ is the Euler function. By Theorem 1.1, we have

$$
F(z, 1 / 2+i T) \ll y^{-1 / 2}+T^{3 / 8+\varepsilon}
$$

Hence, we obtain

$$
\begin{aligned}
F_{\mathfrak{a}}(z, 1 / 2+i T) & \ll \frac{\log q}{(q v)^{1 / 2}} \sum_{\beta \mid v} \sum_{\gamma \mid w} \beta^{1 / 2} \gamma^{-1 / 2}|F(\beta \gamma z, 1 / 2+i T)| \\
& \ll \frac{\log q}{(q v)^{1 / 2}} \sum_{\beta \mid v} \sum_{\gamma \mid w} \beta^{1 / 2} \gamma^{-1 / 2}\left((\beta \gamma y)^{-1 / 2}+T^{3 / 8+\varepsilon}\right) \\
& \ll(q v)^{-1 / 2+\varepsilon} y^{-1 / 2}+q^{-1 / 2+\varepsilon} T^{3 / 8+\varepsilon} .
\end{aligned}
$$

So, we prove (1.12). In particular, if $\mathfrak{a} \sim \infty$, then since $1 / q \sim \infty$ as cusps, we have $v=q$. And, by (6.5), for $y \gg 1 / q$, we obtain

$$
F_{\mathfrak{a}}(z, 1 / 2+i T) \ll q^{-1 / 2+\varepsilon} T^{3 / 8+\varepsilon} .
$$

Now, Theorem 1.2 is proved.

Acknowledgment. The authors would like to thank Professor Jianya Liu for his constant encouragement. They would also like to thank the referee for very useful comments. 


\section{REFERENCES}

[1] C. B. Balogh. Asymptotic expansions of the modified Bessel function of the third kind of imaginary order. SIAM J. Appl. Math., 15:1315-1323, 1967.

[2] V. Blomer, G. Harcos, P. Maga, and D. Milićević. The sup-norm problem for $G L(2)$ over number fields. Arxiv preprint, page 39 pages, 2016.

[3] V. Blomer and R. Holowinsky. Bounding sup-norms of cusp forms of large level. Invent. Math., 179(3):645-681, 2010.

[4] J. B. Conrey and H. Iwaniec. The cubic moment of central values of automorphic $L$-functions. Ann. of Math. (2), 151(3):1175-1216, 2000.

[5] W. Duke, J. B. Friedlander, and H. Iwaniec. The subconvexity problem for Artin $L$-functions. Invent. Math., 149(3):489-577, 2002.

[6] É. Fouvry, E. Kowalski, and P. Michel. Algebraic trace functions over the primes. Duke Math. J., 163(9):1683-1736, 2014.

[7] G. Harcos and N. Templier. On the sup-norm of Maass cusp forms of large level: II. Int. Math. Res. Not. IMRN, 2012(20):4764-4774, 2012.

[8] G. Harcos and N. Templier. On the sup-norm of Maass cusp forms of large level. III. Math. Ann., 356(1):209-216, 2013.

[9] H. Iwaniec. Spectral methods of automorphic forms, volume 53 of Graduate Studies in Mathematics. American Mathematical Society, Providence, RI; Revista Matemática Iberoamericana, Madrid, second edition, 2002.

[10] H. Iwaniec and E. Kowalski. Analytic number theory, volume 53 of American Mathematical Society Colloquium Publications. American Mathematical Society, Providence, RI, 2004.

[11] H. Iwaniec and P. Sarnak. $L^{\infty}$ norms of eigenfunctions of arithmetic surfaces. Ann. of Math. (2), 141(2):301-320, 1995.

[12] S. Ramanujan. Some formulæ in the analytic theory of numbers [Messenger Math. 45 (1916), 81-84]. In Collected papers of Srinivasa Ramanujan, pages 133-135. AMS Chelsea Publ., Providence, RI, 2000.

[13] N. Templier. On the sup-norm of Maass cusp forms of large level. Selecta Math. (N.S.), 16(3):501-531, 2010.

[14] N. Templier. Hybrid sup-norm bounds for Hecke-Maass cusp forms. J. Eur. Math. Soc. (JEMS), 17(8):2069-2082, 2015.

[15] M. Young. A note on the sup norm of Eisenstein series. Arxiv preprint, page 9 pages, 2015.

School of Mathematics, Shandong University, Jinan, Shandong 250100, China

E-mail address: brhuang@mail.sdu.edu.cn

E-mail address: zxu@sdu.edu.cn 